\title{
A NOTE ON THE UNIQUENESS OF RINGS OF COEFFICIENTS IN POLYNOMIAL RINGS
}

\author{
PAUL EAKIN AND K. K. KUBOTA
}

\begin{abstract}
We say that the ring $A$ is of transcendence degree $n$ over its subfield $k$ if for every prime $P \subset A$ the transcendence degree of $A / P$ over $k$ is at most $n$ and equality is attained for some $P$. In this paper we prove the following: Suppose $A$ is a noetherian ring of transcendence degree one over its subfield $k$. Then if $B$ is any ring such that the polynomial rings
\end{abstract}

$$
A\left[X_{1}, \cdots, X_{m}\right] \text { and } B\left[Y_{1}, \cdots, Y_{m}\right]
$$

are isomorphic, $A$ is isomorphic to $B$. Moreover if $A$ has no nontrivial idempotents then either $A$ is isomorphic to the polynomials in one variable over a local artinian ring or, modulo the nil radical, the given isomorphism takes $A$ onto $B$.

This is a continuation of the investigation, begun in [CE] and [AEH], of the extent to which a ring is determined by its polynomial rings. More precisely, we study the following: Suppose $A$ is a ring and $R=A\left[X_{1}, \cdots, X_{n}\right]$ is a ring of polynomials over $A$. If $B$ is a ring and $S=B\left[Y_{1}, \cdots, Y_{n}\right]$ is a ring of polynomials over $B$ such that $R$ is isomorphic to $S$, does it follow that $A$ is isomorphic to $B$ ? In $[\mathbf{C E}]$ and $[\mathbf{A E H}]$ it is shown that for a fairly extensive class of rings $A$, the answer is affirmative. Our main purpose here is to extend this class of rings to include rings of Krull dimension one which are finitely generated over a field. This has been done for the case of rings without zero divisors in [AEH].

Unless there is a statement to the contrary all rings are assumed to be commutative and to possess an identity $1 \neq 0$. If $R$ is a ring, we write $A\left[X_{1}, \cdots, X_{n}\right]=A^{(n)}$ when we want it to be understood or emphasized that the $X_{i}$ 's are algebraically independent over $A$. If $A$ is a ring, we let $N(A)$ denote the nil radical of $A$ and $A^{*}$ the reduced ring $A / N(A)$.

Let $A$ be a ring and $R=A\left[X_{1}, \cdots, X_{n}\right]=A^{(n)}$. We have

$$
N(R)=N(A)\left(R\left[X_{1}, \cdots, X_{n}\right]\right) \text { and } R^{*}=A^{*}\left[\bar{X}_{1}, \cdots, \bar{X}_{n}\right]=A^{*(n)} \text {. }
$$

If $A$ and $B$ are rings and there is an isomorphism

$$
A^{(n)}=A\left[X_{1}, \cdots, X_{n}\right] \stackrel{\sigma}{\longrightarrow} B\left[Y_{1}, \cdots, Y_{n}\right]=B^{(n)},
$$

Received by the editors April 19, 1971.

AMS 1969 subject classifications. Primary 1393, 1320, 1325, 1395, 1420.

Key w'ords and phrases. Polynomial ring, noetherran ring, Krull dimension, affine ring.

(c) American Mathematical Society 1972 
then since $\sigma$ maps the nil radical of $A^{(n)}$ onto the nil radical of $B^{(n)}$ we have an induced isomorphism $\sigma^{*}$ and a commutative diagram with exact rows and columns

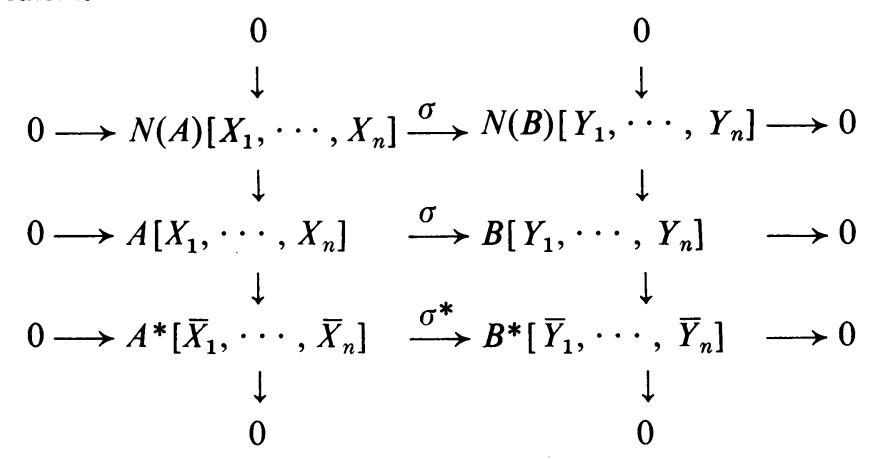

With this notation we give the following:

(1) Definition. A ring $A$ is said to be strongly invariant if for any ring $B$ and any isomorphism of polynomial rings $\sigma: A\left[X_{1}, \cdots, X_{n}\right] \rightarrow$ $B\left[Y_{1}, \cdots, Y_{n}\right]$ we have $\sigma\left(A^{*}\right)=B^{*}$.

A weaker concept is:

(2) Definition. A ring $A$ is said to be invariant if for any ring $B$ such that there is an isomorphism of the polynomial rings $A\left[X_{1}, \cdots, X_{n}\right]$ and $B\left[Y_{1}, \cdots, Y_{n}\right]$, we have $B$ isomorphic to $A$.

Definition (1) is consistent with those of [CE] and [AEH]. In the case where $A$ has no nilpotent elements, and in particular where $A$ is a domain, (1) simply asserts that the isomorphism $\sigma$ takes $A$ onto $B$. With these definitions it is not obvious that strongly invariant rings are invariant. Before proving this we make the following useful observation.

(3) If $A$ is a ring, then $A$ is invariant (resp. strongly invariant) if and only if whenever $B$ is a ring such that $R=A^{(n)}=A\left[X_{1}, \cdots, X_{n}\right]=$ $B\left[Y_{1}, \cdots, Y_{n}\right]=B^{(n)}$ then $A$ is isomorphic to $B$ (resp. $A^{*}=B^{*}$ in $R^{*}$ ).

The proof is straightforward and we omit it.

(4) Strongly invariant rings are invariant.

Proof. Suppose $A$ is a strongly invariant ring. Let $R=A\left[X_{1}, \cdots, X_{n}\right]=$ $A^{(n)}=B\left[Y_{1}, \cdots, Y_{n}\right]=B^{(n)}$ for some $\operatorname{ring} B$, then $A^{*}=B^{*}$ by (3). We claim this implies that $A\left[X_{1}, \cdots, X_{n}\right]=A\left[Y_{1}, \cdots, Y_{n}\right]$. Since $A^{*(n)}=$ $A^{*}\left[\bar{X}_{1}, \cdots, \bar{X}_{n}\right]=A^{*}\left[\bar{Y}_{1}, \cdots, \bar{Y}_{n}\right]$, we have, for each $i, \bar{X}_{i}=$ $\bar{g}_{i}\left(\bar{Y}_{1}, \cdots, \bar{Y}_{n}\right)$, so

$$
X_{i}=g_{i}\left(Y_{1}, \cdots, Y_{n}\right)+n_{i}\left(X_{1}, \cdots, X_{n}\right)
$$

where $n_{i}$ has nilpotent coefficients which are elements of $A$. We also have, for each $i$,

$$
Y_{i}=h_{i}\left(X_{1}, \cdots, X_{n}\right)
$$


Let $\Delta$ be the set of all the coefficients of the $g_{i}, n_{j}$ and $h_{k}$. Set $A_{0}=\Pi[\Delta]$ where $\Pi$ is the prime subring of $A$. Then

$$
A_{0}\left[Y_{1}, \cdots, Y_{n}\right] \subseteq A_{0}\left[X_{1}, \cdots, X_{n}\right]=A_{0}^{(n)}
$$

and

$$
A_{0}^{*}\left[\bar{Y}_{1}, \cdots, \bar{Y}_{n}\right]=A_{0}^{*}\left[\bar{X}_{1}, \cdots, \bar{X}_{n}\right] \text {. }
$$

Now $A_{0}\left[Y_{1}, \cdots, Y_{n}\right]=A_{0}\left[X_{1}, \cdots, X_{n}\right]$. For

$$
A_{0}\left[X_{1}, \cdots, X_{n}\right] \subseteq A_{0}\left[Y_{1}, \cdots, Y_{n}\right]+N\left(A_{0}\right)\left[Y_{1}, \cdots, Y_{n}\right],
$$

and iterating this we have

$$
A_{0}\left[X_{1}, \cdots, X_{n}\right] \subseteq \sum_{j=0}^{k-1}\left[N\left(A_{0}\right)\right]^{j}\left[Y_{1}, \cdots, Y_{n}\right]
$$

Since $A_{0}$ is noetherian, there is a $k$ such that $\left[N\left(A_{0}\right)\right]^{k}=0$, and therefore $A\left[X_{1}, \cdots, X_{n}\right]=A\left[Y_{1}, \cdots, Y_{n}\right]$. Now we claim that the $Y_{i}$ are algebraically independent over $A$. For if $c\left(Y_{1}, \cdots, Y_{n}\right)=0$ is an equation of algebraic dependence and $A_{1}$ is the subring of $A$ generated by $A_{0}$ and the coefficients of $c$, we have

$$
A_{1}\left[X_{1}, \cdots, X_{n}\right]=A_{1}\left[Y_{1}, \cdots, Y_{n}\right] .
$$

Since the $X_{i}$ are algebraically independent over $A_{1}$ there is an $A_{1}$-homomorphism

$$
A_{1}\left[X_{1}, \cdots, X_{n}\right] \stackrel{\tau}{\longrightarrow} A_{1}\left[Y_{1}, \cdots, Y_{n}\right]
$$

which takes $X_{i}$ to $Y_{i}$. Then $c\left(X_{1}, \cdots, X_{n}\right)$ must be in the kernel of $\tau$. Since $A_{1}\left[Y_{1}, \cdots, Y_{n}\right]=A_{1}\left[X_{1}, \cdots, X_{n}\right], \tau$ is a homomorphism of $A_{1}\left[X_{1}, \cdots, X_{n}\right]$ onto itself. Since any homomorphism of a noetherian ring onto itself is an isomorphism, $\tau$ is an isomorphism and hence all of the coefficients of $c$ are zero. Thus the $Y$ 's are algebraically independent over $A$. Now we have immediately that $A$ is isomorphic to $B$, for if $\mathfrak{A}$ is the ideal of $R$ generated by $\left\{Y_{1}, \cdots, Y_{n}\right\}$, then $R / \mathfrak{A}$ is isomorphic to both $A$ and $B$. This concludes the proof of (3).

In $[\mathbf{A E H}]$ the following is proved:

If $A$ is a domain which is of transcendence degree one over a field, then $A$ is invariant. $A$ is either strongly invariant or there is a field $k$ such that $A=k[t]=k^{(1)}$.

In the sequel any reference to $[\mathbf{A E H}]$ is an appeal to this result. This shows that if $t$ is transcendental over the field $k$, then $k[t]$ is an invariant ring which is not strongly invariant, for $k[t][X]=k[X][t]$ but $k[t] \neq k[X]$.

(5) REMARK. We do not know of an example of a commutative ring with identity which is not invariant. However there are simple examples 
of noninvariant rings without identity. In fact let $G$ be any abelian group such that $G$ is not isomorphic to $G \oplus G$. Make each of these groups into a ring defining the product of any two elements to be zero. Then $G[X]$ and $(G \oplus G)[Y]$ are each isomorphic to a direct sum of countably many copies of $G$, hence they are isomorphic.

(6) If $A$ is a noetherian ring then $A$ is invariant if and only if whenever

$$
A^{(n)}=A\left[X_{1}, \cdots, X_{n}\right]=B\left[Y_{1}, \cdots, Y_{n}\right]=B^{(n)},
$$

there is a homomorphism from $A$ onto $B$.

Proof. Such a homomorphism can be extended to a mapping of $A\left[X_{1}, \cdots, X_{n}\right]$ onto $B\left[Y_{1}, \cdots, Y_{n}\right]$ which would be a homomorphism of $A\left[X_{1}, \cdots, X_{n}\right]$ onto itself. Since any onto endomorphism of a noetherian ring is an isomorphism, the original homomorphism must have been an isomorphism.

(7) Any finite direct sum of invariant rings is invariant; any finite direct sum of strongly invariant rings is strongly invariant.

Proof. Let $A=A_{1} \oplus \cdots \oplus A_{k}$ and

$$
A^{(n)}=A\left[X_{1}, \cdots, X_{n}\right]=B\left[Y_{1}, \cdots, Y_{n}\right]=B^{(n)}=R,
$$

and let $e_{i}$ be the identity of $A_{i}$. Since $e_{i}$ is idempotent, it is easy to see that $e_{i} \in B$ for each $i$. Thus $B e_{1}+B e_{2}+\cdots+B e_{k}$ is a direct sum decomposition of $B$ and $A e_{i}\left[e_{i} X_{1}, \cdots, e_{i} X_{n}\right]=B e_{i}\left[e_{i} Y_{1}, \cdots, e_{i} Y_{n}\right]=e_{i} R$ becomes

$$
A_{i}^{(n)}=A_{i}\left[\bar{X}_{1}, \cdots, \bar{X}_{n}\right]=B_{i}\left[\bar{Y}_{1}, \cdots, \bar{Y}_{n}\right]=B_{i}^{(n)} .
$$

If each $A_{i}$ is strongly invariant then $A_{i}$ is isomorphic to $B_{i}$ for each $i$ and consequently $A$ is isomorphic to $B$.

We say that the ring $R$ is of transcendence degree $n$ over a field $F$ if $R$ contains $F, \operatorname{deg} \operatorname{tr}_{F} R / p \leqq n$ for each prime ideal $p \subseteq R$, and there is a prime ideal $p$ for which equality is attained.

(8) Let $A$ be a one dimensional noetherian ring which is of transcendence degree one over a field and suppose $A$ has no nontrivial idempotents. Then if $A$ has at least two primes of height zero, $A$ is strongly invariant.

Proof. Let $A^{(n)}=A\left[X_{1}, \cdots, X_{n}\right]=B\left[Y_{1}, \cdots, Y_{n}\right]=B^{(n)}$. We wish to show $A^{*}=B^{*}$. Let $q_{1}, \cdots, q_{k}$ be the height zero primes of $A$. Then since $\mathfrak{A}_{i}=q_{i}\left[X_{1}, \cdots, X_{n}\right]$ is also of height zero, there are primes $p_{1}, \cdots, p_{k}$ of $B$ such that $p_{i}\left[Y_{1}, \cdots, Y_{n}\right]=q_{i}\left[X_{1}, \cdots, X_{n}\right]$ and the $p_{i}$ are precisely the height zero primes of $B$. Thus we have a diagram:

$$
\begin{gathered}
A\left[X_{1}, \cdots, X_{n}\right]=B\left[Y_{1}, \cdots, Y_{n}\right]=R, \\
\downarrow \\
A / q_{i}\left[\bar{X}_{1}, \cdots, \bar{X}_{n}\right]=B / p_{i}\left[\bar{Y}_{1}, \cdots, \bar{Y}_{n}\right]=R / \mathfrak{I}_{i}
\end{gathered}
$$

and, to see that $A^{*}=B^{*}$, it is sufficient to see that $A / q_{i}=B \mid p_{i}$ in $R / \mathfrak{R}_{i}$ for 
each $i$. Since $A$ has no nontrivial idempotents, $A$ cannot be represented as a direct sum and thus $q_{1}$ and $b=\prod_{i=2}^{k} q_{i}$ are contained in a common maximal ideal. For if not we would have $A=A / q_{1}^{t} \oplus A / b^{t}$ whenever $(N(A))^{t}=0$. Let $M$ be a maximal ideal of $A$ such that $q_{1}+b \leqq M$. Since $M$ is prime, at least one of the $q_{i}(i \neq 1)$ is contained in $M$, say $q_{2} \subset M$. Now $q_{1}+q_{2} \leqq M$ implies $q_{1}\left[X_{1}, \cdots, X_{n}\right], q_{2}\left[X_{1}, \cdots, X_{n}\right] \subseteq M\left[X_{1}, \cdots, X_{n}\right]$, and therefore $p_{1}+p_{2} \subseteq M\left[X_{1}, \cdots, X_{n}\right] \cap B=N$. Since $p_{1}$ and $p_{2}$ are distinct, $p_{1}+p_{2}$ is not of height zero and therefore $N$ is a maximal ideal of $B$ such that $N\left[Y_{1}, \cdots, Y_{n}\right]=M\left[X_{1}, \cdots, X_{n}\right]$. Now in

$$
R_{1}=A / q_{1}\left[\bar{X}_{1}, \cdots, \bar{X}_{n}\right]=\left(A / q_{1}\right)^{(n)}=B / p_{1}\left[\bar{Y}_{1}, \cdots, \bar{Y}_{n}\right]=\left(B / p_{1}\right)^{(n)}
$$

we have that either $A / q_{1}=B / p_{1}$ or each is of the form $k[T]=k^{(1)}$ for some field $k$ [AEH]. But even in the latter case, they are equal. For each is a P.I.D., so set $M / q_{1}=(a)$ and $N / p_{1}=(b)$. Then $a R_{1}=b R_{1}$ and so $a$ and $b$ differ only by a unit of $R_{1}$. Thus $a \in A / q_{1} \cap B / p_{1}$. Since $a$ generates a nonzero prime of $A / q_{1}$, it is transcendental over $k$. Thus it is a transcendence base for both $A / q_{1}$ and $B / p_{1}$ over $k$. But each of $A / q_{1}$ and $B / p_{1}$ is the algebraic closure of $k[a]$ in $R_{1}$, and hence $A / q_{1}=B / p_{1}$. Since we could have chosen any prime of height zero to be $q_{1}$, it follows that $A^{*}=B^{*}$ and we have completed the argument.

(9) REMARK. In case $A$ is a reduced affine ring over a field, the previous argument has a geometric interpretation. One views $A$ as the coordinate algebra of an affine curve $\Gamma_{A}$. Then $A[X]$ is the coordinate algebra of the cylinder $C_{A}$ over $\Gamma_{A}$ (see Figure 1).

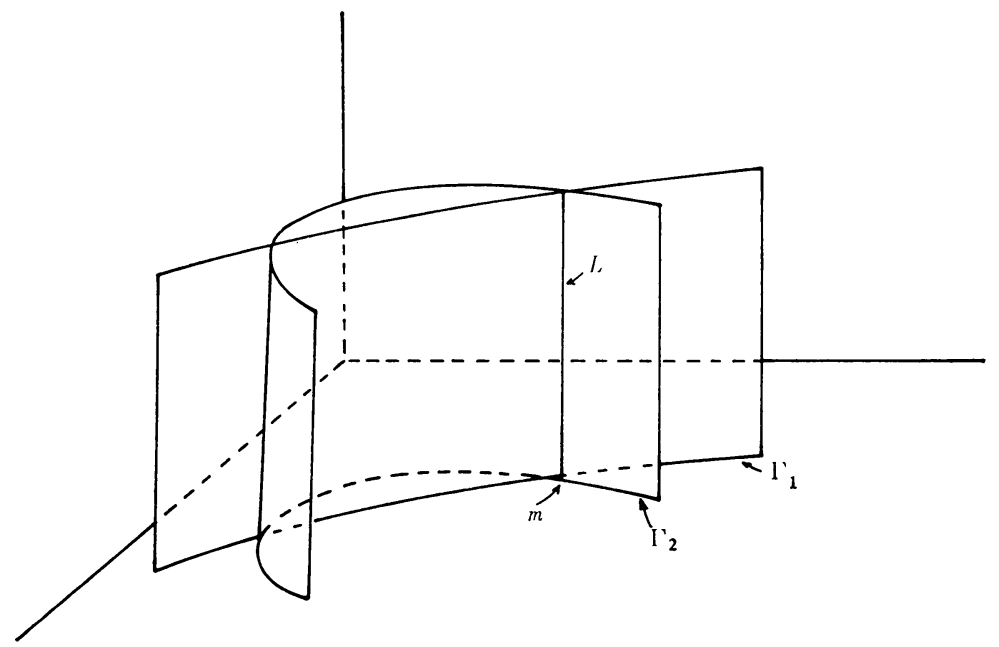

FIGURE 1 
If $\Gamma_{A}$ is not irreducible, then it is the union of a finite number of irreducible components $\Gamma_{1}, \cdots, \Gamma_{k}$ where the $\Gamma_{i}$ 's correspond to the height zero primes $q_{i}$. The assumption that $A$ has no nontrivial idempotents is equivalent to the fact that our curve $\Gamma_{A}$ is connected in the Zariski topology. In the proof of (8) the maximal ideal $M$ such $q_{1}+q_{2} \subseteq M$ corresponds to a point $m$ on the intersection of the components $\Gamma_{1}$ and $\Gamma_{2}$. The assumption $A[X] \cong B[Y]$ corresponds to the existence of a biregular mapping $\phi$ from $C_{A}$ onto a cylinder $C_{B}$ over some curve $\Gamma_{B}$. With reference to Figure 1 the proof essentially shows that lines such as $L$ on $C_{A}$ ( $L$ corresponds to $M[X]$ ) must be taken to similar lines on $C_{B}$ by $\phi$ (our statement $M[X]=N[Y])$. The proof then can be interpreted as saying that the necessity of lines such as $L$ going onto similar lines imparts so much rigidity that the biregular mapping must essentially take $\Gamma_{A}$ onto $\Gamma_{B}$.

(10) Let $A$ be a noetherian ring of transcendence degree one over a field and suppose $A$ has a unique minimal prime $p$ such that $A / p=k\left[t^{\prime}\right]=$ $k^{(1)}$. Then $A$ is invariant. Moreover the following conditions are equivalent:

(1) $A$ is strongly invariant.

(2) $A$ is not of the form $A_{0}[X]=A_{0}^{(1)}$ where $A_{0}$ is a local artinian ring.

(3) $A$ has an embedded prime divisor of (0).

Proof. First we show the equivalence of conditions (1), (2) and (3) above.

(3) $\Rightarrow(1)$. Let $p$ be the unique minimal prime of $A$. We must show that if $R=A^{(n)}=A\left[X_{1}, \cdots, X_{n}\right]=B\left[Y_{1}, \cdots, Y_{n}\right]=B^{(n)}$ then $A^{*}=B^{*}$. Let $M$ be an embedded prime divisor of zero in $A$. Then $M$ is maximal, so there is a maximal ideal $N \subset B$ such that $N\left[Y_{1}, \cdots, Y_{n}\right]=M\left[X_{1}, \cdots, X_{n}\right]$. This is because $M\left[X_{1}, \cdots, X_{n}\right]$ is an associated prime of $(0)$ in $R$. For if $N_{1}, \cdots, N_{k}$ are the associated primes of $(0)$ in $B$, then $\left\{N_{i} R\right\}_{i=1}^{k}$ are the primes of (0) in $R$. By the uniqueness of these primes, $M\left[X_{1}, \cdots, X_{n}\right]=$ $N_{i}\left[Y_{1}, \cdots, Y_{n}\right]$ for some $i$. Now we go to

$$
A^{*(n)}=A^{*}\left[\bar{X}_{1}, \cdots, \bar{X}_{n}\right]=B^{*}\left[\bar{Y}_{1}, \cdots, \bar{Y}_{n}\right]=B^{*(n)}
$$

and use almost exactly the same argument which concludes (8).

$(1) \Rightarrow(2)$. This is clear for $A_{0}[X]=A_{0}^{(1)}$ is not strongly invariant since $A_{0}[X][Y]=A_{0}[Y][X]$.

(2) $\Rightarrow(3)$. We must show that if $A$ has no embedded prime divisors of (0), then $A$ is of the form $A_{0}[X]=A_{0}^{(1)}$. Let

$$
A \stackrel{\sigma}{\rightarrow} A \mid p=k\left[t^{\prime}\right]=k^{(1)}
$$

be the natural map and let $I=\sigma^{-1}(k)$. Since $p$ is the nil radical of $I, I$ is a local ring with maximal ideal $p$. Thus $I$ is a complete local ring. By Cohen's structure theorem of complete local rings [ZS, Theorem 27, 
p. 304], I contains a field $k_{0}$ which is naturally isomorphic to $k$. Thus $k_{0} \subseteq A$ and $\sigma\left(k_{0}\right)=k$. We now identify $k_{0}$ and $k$.

Choose a preimage $t$ of $t^{\prime}$ in $A$. If $\lambda_{1}, \cdots, \lambda_{r}$ generate $p$, we have $k\left[\lambda_{1}, \cdots, \lambda_{r}\right][t]=A$. The element $t$ is, in fact, transcendental over $k\left[\lambda_{1}, \cdots, \lambda_{r}\right]=A_{0}$. For consider the homomorphism

$$
k\left[\lambda_{1}, \cdots, \lambda_{r}\right][X] \stackrel{\phi}{\longrightarrow} k\left[\lambda_{1}, \cdots, \lambda_{r}\right][t]=A .
$$

We claim this is an isomorphism. Let $K=\operatorname{kernel} \phi$. Then $K$ must be contained in $N\left(A_{0}\right)[X]=\left(\lambda_{1}, \cdots, \lambda_{r}\right)[X]$. If not, it would follow that there is a $j(t) \subseteq p$ where $j$ is a nonzero polynomial with coefficients in $k$. But since $A \mid p=k\left[t^{\prime}\right]$ and $t^{\prime}$ is transcendental over $k$, this is impossible. We suppose there is a least integer $m$ such that the kernel of the map $\sigma^{\prime \prime}$ in the following commutative diagram is zero. Here the rows are exact.

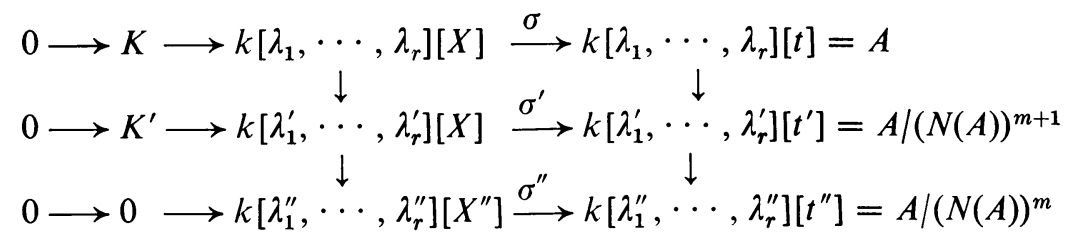

We claim $K^{\prime}=0$ (for convenience we assume $N(A)^{m+1}=0$ ). Now $N(A)^{m}$ is a finite module over $A / N(A)=k\left[t^{\prime}\right]$. Moreover this is a torsion free module since if for some $n \in N(A)^{m}, f \in k\left[t^{\prime}\right], f n=0$ then $f(t)$ would be a zero divisor in $A$. However $A$ has no embedded primes of zero, and it follows that $f(t) \in p$. This implies $f\left(t^{\prime}\right)=0$. Thus there is a free basis for $N(A)^{m}$ over $A / N(A)$, say $\xi_{1}, \cdots, \xi_{s}$. Let $h \in K^{\prime}$. Then $h=\sum \xi_{i} h_{i}(X)$ by the minimality of $m$. But $\sigma^{\prime}(h)=\sum \xi_{i} h_{i}\left(t^{\prime}\right)=0$ implies $h_{i}=0$ for each $i$ and thus $h=0$. Thus $\sigma^{\prime}$ is an isomorphism in contradiction of the minimality of $m$. It follows then that $\sigma$ is an isomorphism modulo every power of $N(A)$. But for a suitably high power, this is zero. Thus $\sigma$ is an isomorphism, and hence $(2) \Rightarrow(3)$.

To complete our proof we must show that $A_{0}[X]=A_{0}^{(1)}$ is invariant if $A_{0}$ is an artinian local ring. Suppose $A_{0}=k\left[\lambda_{1}, \cdots, \lambda_{r}\right]$ where the $\lambda_{i}$ are nilpotent and let $A=A_{0}[X]$. If $R=A^{(n)}=A\left[X_{1}, \cdots, X_{n}\right]=B\left[Y_{1}, \cdots, Y_{n}\right]=$ $B^{(n)}$, then we may assume $B^{*}=k\left[t^{\prime}\right]=k^{(1)}$ since $k[X]$ is invariant [AEH]. Let $t$ be a preimage of $t^{\prime}$ and

$$
\lambda_{i}=\eta_{i}+\sum Y_{i} g_{i}\left(Y_{1}, \cdots, Y_{n}\right) \in B\left[Y_{1}, \cdots, Y_{n}\right]
$$

Then since $N(R)=\left(\lambda_{1}, \cdots, \lambda_{r}\right) R$ it follows that $\left\{\eta_{1}, \cdots, \eta_{r}\right\}$ generates $N(B)$ and $B=k\left[\eta_{1}, \cdots, \eta_{r}, t\right]$. If $h$ is a polynomial with coefficients in 
$k$ and $h\left(\lambda_{1}, \cdots, \lambda_{r}\right)=0$, then substituting from $(*)$ we see $h\left(\eta_{1}, \cdots, \eta_{r}\right)=$ 0 . Thus there is a well defined $k$-homomorphism $k\left[\lambda_{1}, \cdots, \lambda_{r}\right] \rightarrow$ $k\left[\eta_{1}, \cdots, \eta_{r}\right]$ which takes $\lambda_{i} \rightarrow \eta_{i}$. This can be extended to a homomorphism of $A$ onto $B$. Thus $A$ is invariant by (6).

(11) THEOREM. If $A$ is a one dimensional affine ring over a field $k$, then $A$ is invariant. Moreover $A$ is strongly invariant unless $A$ can be expressed in the form $A_{1} \oplus A_{0}[X]$ where $A_{0}$ is a local artinian ring and $X$ is an indeterminate over $A_{0}$.

Proof. Let $e_{1}, \cdots, e_{r}$ be a maximal set of pairwise orthogonal idempotents of $A$. Then $A=A e_{1} \oplus \cdots \oplus A e_{r}$. Now apply (7), (8) and (10) to complete the argument.

(12) Remark. Our Definitions (1) and (2) of invariant and strongly invariant could be taken to define $n$-invariant and $n$-strongly invariant where $n$ is the number of variables. We do not know if it is possible for a ring to be $n$-invariant and not $m$-invariant for different integers $m$ and $n$. In particular does 1 -invariant imply $n$-invariant?

We close with an example of a strongly invariant, one dimensional affine ring $A$ such that there is an isomorphism of polynomial rings $\sigma: A\left[X_{1}, \cdots, X_{n}\right] \rightarrow B\left[Y_{1}, \cdots, Y_{n}\right]$, yet $\sigma(A) \neq B$. Let $k$ be a field of characteristic 2 and let $A=k\left[a^{2}, a^{3}, \theta\right] /\left(\theta^{2}\right)$. If $A^{(n)}=A\left[X_{1}, \cdots, X_{n}\right]=$ $B\left[Y_{1}, \cdots, Y_{n}\right]=B^{(n)}$ for some $\operatorname{ring} B$, then $A^{*}=B^{*}$ since $A^{*}=k\left[a^{2}, a^{3}\right]$ is a strongly invariant ring [AEH]. Now let $X$ be an indeterminate over $A$ and set $B=k\left[a^{2}, a^{3}+\theta X, \theta\right]$. Then $A[X]=B[X]$ and $A \neq B$. However we must show that $X$ is an indeterminate over $B$. If not, suppose $b_{0}+$ $b_{1} X+\cdots+b_{s} X^{s}=0$ with $b_{i} \in B$. Then since $A^{*}=B^{*}$, we must have $\theta$ divides each $b_{i}$, say $b_{i}=\theta g_{i}\left(a^{2}, a^{3}+\theta X, \theta\right)$. We have then

$$
0=\sum_{i=0}^{s} \theta g_{i}\left(a^{2}, a^{3}+\theta X, \theta\right) X^{i}=\sum_{i=0}^{s} \theta g_{i}\left(a^{2}, a^{3}, \theta\right) X^{i}
$$

since $\theta^{2}=0$. Hence $\theta g_{i}\left(a^{2}, a^{3}, 0\right)=0$ for each $i$ since $X$ is transcendental over $A$. Therefore $g_{i}(T, Y) \equiv 0 \bmod \left(T^{3}-Y^{2}\right)$ so

so

$$
g_{i}(T, Y, Z)=\left(T^{3}-Y^{2}\right) h(T, Y, Z)+Z q(T, Y, Z)
$$

$$
g_{i}\left(a^{2}, a^{3}+\theta X, \theta\right)=\left[\left(a^{2}\right)^{3}-\left(a^{3}+\theta X\right)^{2}\right] h+\theta q=\theta q .
$$

Hence $b_{i}=\theta g_{i}\left(a^{2}, a^{3}+\theta X, \theta\right)=\theta^{2} q=0$ which shows $X$ is transcendental over $B$.

ADDED IN PROOF. M. Hochster has recently given an elegant example of an integral domain which is not invariant. Hochster's example is a four dimensional affine ring over the field of real numbers. 


\section{REFERENCES}

[AEH] S. Abhyankhar, P. Eakin and W. Heinzer, On the uniqueness of the coefficient ring in a ring of polynomials, J. Algebra (to appear).

[CE] D. Coleman and E. Enochs, Isomorphic polynomial rings, Proc. Amer. Math. Soc. 27 (1971), 247-252.

[ZS] O. Zariski and P. Samuel, Commutative algebra. Vol. 2, The University Series in Higher Math., Van Nostrand, Princeton, N.J., 1960. MR 22 \#11006.

Department of Mathematics, University of Kentucky, Lexington, Kentucky 40506 